\title{
Tacrolimus-associated Pruritus: Role of a Clinical Pharmacologist in the Management
}

Saibal Das ${ }^{1}$, Saptarshi Bishnu ${ }^{2}$

Bengal Physician Journal (2020): 10.5005/jp-journals-10070-7028

Sir,

We read the case report on tacrolimus-associated pruritus by Sen et al. and was intrigued by the suggestions by the authors of clinical pharmacological consultation in cases of suspected drugrelated problems. ${ }^{1}$ A 46-year-old male with comorbidities like type 2 diabetes and hypertension underwent $A B O$-incompatible liver transplantation in January 2020. He was on multiple drugs, such as basal-bolus insulin (long-acting glargine insulin and rapid-acting insulin aspart), tacrolimus $3 \mathrm{mg}$ twice daily, prednisolone $10 \mathrm{mg}$ twice daily, mycophenolate mofetil $250 \mathrm{mg}$ once daily, amlodipine $10 \mathrm{mg}$ once daily, and ursodeoxycholic acid $300 \mathrm{mg}$ twice daily. The patient visited a dermatologist with a complaint of generalized itching since March 2020. He was managed conservatively with anti-allergic drugs and emollient. However, the clinical condition did not improve. Liver function test showed normal serum bilirubin, alanine transaminase, aspartate transaminase, and alkaline phosphatase levels. Magnetic resonance cholangiopancreatography did not reveal any significant dilatation of the intrahepatic bile ducts, although small perihepatic fluid pockets were seen. Besides, the blood glucose level was fluctuating, and there were several episodes of postprandial hyperglycemia (glucose level, $350-400 \mathrm{mg} / \mathrm{dL}$ ) with evening and night-time hypoglycemic episodes (glucose levels, 40-70 mg/dL). Itching intensified with localized skin swelling around the insulin administration site since early June 2020. The liver function test was found to be within the normal limit during that time period.

The patient went for a consultation with a clinical pharmacologist via telemedicine. The occurrence of an error in insulin dosing and administration time was noted - the patient took glargine insulin thrice daily instead of rapid-acting aspart insulin. The same was rectified; however, the itching persisted. The blood level of tacrolimus was towards the higher therapeutic range (14 mg/dL). The liver and renal functions were within normal limits. The clinical pharmacological advisory suggested replacing tacrolimus with everolimus, along with a consultation with the liver transplantation physician. Compliance with the advice was rewarded with the resolution of symptoms and resumption of glycemic control in 2 weeks.

The success of solid organ transplantation (SOT) depends, in part, on immunosuppressive therapy. ${ }^{2,3}$ Tacrolimus is a calcineurin inhibitor (CNI) and macrolide antibiotic that is frequently used as an immunosuppressant following solid organ transplantation. It
${ }^{1}$ Independent Clinical Pharmacology Consultant, Kolkata, West Bengal, India

${ }^{2}$ Consultant Hepatologist and Liver Transplant Physician, Apollo Hospital, Chennai, Tamil Nadu, India

Corresponding Author: Saibal Das, Independent Clinical Pharmacology Consultant, Kolkata, West Bengal, India, Phone: +91 9433429401, e-mail: saibaldas123@gmail.com

How to cite this article: Das S, Bishnu S. Tacrolimus-associated Pruritus: Role of a Clinical Pharmacologist in the Management. Bengal Physician Journal 2020;7(2):48-49.

Source of support: Nil

Conflict of interest: None

inhibits interleukin-2-mediated cell proliferation. Its therapeutic window is narrow. ${ }^{4,5} \mathrm{CNIs}$ lead to long-term complications, such as nephrotoxicity and increased risk for hepatocellular carcinoma recurrence. ${ }^{6-8}$ This warrants an alternative approach to minimize chronic $\mathrm{CNI}$ exposure, for example, the use of mammalian target of rapamycin (mTOR) inhibitor, such as everolimus. $^{8}$

The problems associated with this case were of two types. One was drug administration error related to both long-and rapid-acting insulin, which was promptly solved. The other problem of generalized itching was solved with the discontinuation of tacrolimus and switching to everolimus. Although everolimus is structurally similar to tacrolimus, it has a different mechanism of action (mTOR inhibitor). ${ }^{9}$ This case highlights the importance of a clinical pharmacologist consultation in managing drug-related problems in routine patient care. The opinion of a clinical pharmacologist can be routinely used to ensure a favorable clinical outcome.

\section{References}

1. Sen S, Tripathi SK, Samajdar SS. Tacrolimus-associated pruritus: case report and review. Bengal Phys J 2020;7(1):12-13.

2. Black CK, Termanini KM, Aguirre O, et al. Solid organ transplantation in the 21st century. Ann Transl Med 2018;6(20):409. DOI: 10.21037/ atm.2018.09.68.

3. Rodriguez-Peralvarez M, De la Mata M, Burroughs AK. Liver transplantation: immunosuppression and oncology. Curr Opin Organ Transplant 2014;19(3):253-260. DOI: 10.1097/MOT.0000000000000069.

4. Pennington CA, Park JM. Sublingual tacrolimus as an alternative to oral administration for solid organ transplant recipients. Am J Health Syst Pharm 2015;72(4):277-284. DOI: 10.2146/ajhp140322.

\footnotetext{
(c) The Author(s). 2020 Open Access This article is distributed under the terms of the Creative Commons Attribution 4.0 International License (https:// creativecommons.org/licenses/by-nc/4.0/), which permits unrestricted use, distribution, and non-commercial reproduction in any medium, provided you give appropriate credit to the original author(s) and the source, provide a link to the Creative Commons license, and indicate if changes were made. The Creative Commons Public Domain Dedication waiver (http://creativecommons.org/publicdomain/zero/1.0/) applies to the data made available in this article, unless otherwise stated.
} 
5. Farkas SA, Schnitzbauer AA, Kirchner G, et al. Calcineurin inhibitor minimization protocols in liver transplantation. Transpl Int 2009;22(1):49-60. DOI: 10.1111/j.1432-2277.2008.00796.x.

6. Mihatsch MJ, Kyo M, Morozumi K, et al. The side-effects of ciclosporine-A and tacrolimus. Clin Nephrol 1998;49(6):356-363.

7. Nankivell BJ, BorrowRJ,Fung CL, etal.Calcineurininhibitornephrotoxicity: longitudinal assessment by protocol histology. Transplantation 2004;78(4):557-565. DOI: 10.1097/01.tp.0000128636.70499.6e.
8. Wei KC, Lai PC. Combination of everolimus and tacrolimus: a potentially effective regimen for recalcitrant psoriasis. Dermatol Ther 2015;28(1):25-27. DOI: 10.1111/dth.12176.

9. Reitamo S, Spuls P, Sassolas B, et al. Efficacy of sirolimus (rapamycin) administered concomitantly with a subtherapeutic dose of cyclosporin in the treatment of severe psoriasis: a randomized controlled trial. Br J Dermatol 2001;145(3):438-445. DOI: 10.1046/j.1365-2133.2001. 04376.x. 